diagnosis "stroke". Almost as many men $(n=46)$ as women ( $n=47$ ) were treated. The average age of the patients was 77 years, while the most strokes were observed in the age group ranging from 80 to 90 years $(n=42)$. Not only the diastolic, but also the systolic blood pressure was to be measured in at least 10 -minute intervals. The average systolic blood pressure was $154 \mathrm{mmHg}$, while 3 patients had systolic pressures of $<100 \mathrm{mmHg}$ and 16 patients $>190$ $\mathrm{mmHg}$. The diastolic blood pressure was around 85.5 $\mathrm{mmHg}$. The body temperature was to be taken axillary with digital thermometers. 61 patients did not run a fever, 7 patients had a slightly higher temperature, and 2 patients had a temperature exceeding $38.0^{\circ} \mathrm{C}$.

The oxygen saturation amounted to an average of $94.9 \%$. Yet 6 patients had a primary oxygen saturation of less than $90 \%$.

Key words: algorithm; oxygen saturation; prehospital; protocol; risk factors; stroke

Prehosp Disast Med 2001;16(2):s85.

\section{Remote Area Mass Casualty Incident Response in Australia \\ Eric Williams \\ AUSTRALIA}

Australia has a land mass of 7,682,300 sq. km., approximately the same size as the United States of America excluding Alaska, 32 times the size of the United Kingdom, twice the size of Europe (excluding the Russian Federation), and three to four times the size of the European Union (European Economic Community). Australia's population of 20 million is mostly centred around state capital cities which are situated peripherally on the island continent. Distance is a major consideration for planning a response to mass casualty incidents. Under the Australian Constitution, the Federal Government of The Commonwealth of Australia has the responsibility for the defence of Australia, and for protection of the States against invasion. However, each state and territory is responsible for the protection of citizens and property, including emergency planning and response within its jurisdiction. The Commonwealth centrally, plans and coordinates programs for response to national threats affecting national interests and provides coordination of actions between states if required. Such national programs include the development of a cyclone warning system and the Commonwealth Search and Rescue Organization, which coordinates the initial response to air and maritime incidents.

This paper outlines the measures necessary to provide effective mass casualty management for remote area incidents and for communities, which may be remote from major treatment centres. The medical coordination and organization issues involved in prehospital management and the distribution of casualties to appropriate treatment facilities will be outlined.

Key words: Australia; cyclone; mass casualties; maritime; plan; preparedness; remote; responses; responsibility; search and rescue

Prebosp Disast Med 2001;16(2):s85.

\section{Distal Tubes for Left Colorectal Trauma and Obstructive Carcinomas \\ Wu Yinai; Liu Xiantang; Xie Shangkui; et al}

Department of General Surgery, The 157th Central Hospital of PLA, Guangzhou, CHINA

Objective: To prevent and reduce anastomotic leakage after a one-stage operation for left colorectal trauma and obstructive carcinomas, and after Dixon operation for rectal carcinomas.

Methods: Drainage by perianastomotic, proximal and distal tubes was used in 142 patients with left obstructive colonic carcinomas, 86 patients with left colorectal trauma, and 157 patients undergoing a Dixon operation. The incidence of postoperative anastomotic leakage and the healing time of lower anastomotic leakage after operation for rectal carcinomas were observed.

Results: By means of drainage by perianastomotic proximal and distal tubes, the incidence of postoperative anastomotic leakage was $4.2 \%(6 / 142)$ and $1.9 \%(3 / 157)$ respectively in patients with left obstructive colorectal carcinomas and patients undergoing Dixon operation for rectal carcinomas. These indexes were different from those in control groups $(p<0.05)$. The incidence of postoperative anastomotic leakage was $3.5 \%(3 / 86)$ in patients with left colorectal trauma.

Conclusion: Drainage by perianastomotic proximal and distal tubes can effectively prevent and reduce the incidence of anastomotic leakage after one-stage operation for left colorectal trauma, obstructive carcinomas, and after Dixon operation for rectal carcinomas.

Key words: carcinoma; colorectal trauma; leakage; operations; perianastomotic proximal and distal tubes; surgery Prebosp Disast Med 2001;16(2):s85.

\section{Prediction of Death in Patients with Acute Hypertensive Cerebral Hemorrhage Wu Zbenhong}

Guangxi National Hospital, Guangxi Nanning, PEOPLE'S REPUBLIC OF CHINA

Objective: In order to increase the rate of success of emergency treatment for patients with an acute, hypertensive cerebral hemorrhage, the risk factors for predicting death were studied in 101 patients.

Methods: The relationships between age, state of consciousness, blood pressure (BP) at the onset, amount of bleeding; and morality were analyzed retrospectively.

Results: The older the patient; the worse the state of consciousness, the higher the BP at the onset, the higher the amount of bleeding, and the higher the mortality $(p<0.01$.) Conclusion: The aforementioned indices are of prognostic significance for patients with an acute, hypertensive cerebral hemorrhage.

Key words: cerebral hemorrhage; consciousness; hypertension; mortality; prediction; stroke Prehosp Disast Med 2001;16(2):s85. 